Signal \& Image Processing : An International Journal (SIPIJ) Vol.3, No.4, August 2012

\title{
IMAGE PROCESSING OF LIQUID CRYSTAL MESOGEN EXHIBITING NEMATIC PHASE
}

\author{
Ratna Raju.M ${ }^{1}$, Ch.RaviShankar Kumar ${ }^{2}$ \\ ${ }^{1}$ Faculty, Dept. of Physics, A.C.College Guntur, India 522004 \\ ${ }^{2}$ Faculty, Dept. of Physics, Institute of Science, GITAM University India 530045 \\ rskchavalegmail.com
}

\begin{abstract}
Analysis of textures is performed with finite number of grains in exploring the statistical properties that are obtained experimentally and analyzed for gray level coocurrence features. The article attempts novel method in analysis of liquid crystalline mesogen exhibiting nematic texture with image processing techniques for variety of features and its relevance to the texture quantitatively. The texture possesses orientation property with rod like molecules along the director where the centre of symmetry coincides with centre of gravity. The nematic texture is obtained for p-azozyanisole is thermotropic liquid crystal which is monotropic that experience the same texture in both heating and cooling process. The results of measurements reveal that it has minimum energy associated with clusters formed during the transition with low energy and entropy responsible for first order transition along with relevant attributes responsible for texture in the entire process.
\end{abstract}

\section{KEYWORDS:}

Liquid crystal, Nematic phase, Texture and Matlab

\section{INTRODUCTION}

Liquid crystals [1-2] reflect the characteristic textures that vary with temperature and concentration termed as thermotropic and later as lyotropic liquid crystals. These molecules that are innumerable in number with different spatial organizations shear due to its anisotropic nature with temperature are considered. The packing of these molecules change due to thermal energy giving rise to different liquid crystalline textures[3-4] .An attempt is made on these textures with image processing techniques[5-8] exploring the gray level coocurence[9-11] features. Generally speaking texture refers to repetition of pattern that is complex with number of units termed as pixels with units and subunits described with coarse, grained, smooth, brightness, color, shape, size etc and further change in different regions. Texture classification is concerned with identifying different regions from set of textures and segmentation is concerned with boundaries between various regions of texture in an image. These textures discriminate between different regions, produce descriptions and to segment an image. Analysis of these textures are performed with mathematical method involving statistical procedures[9] that determine the variations in image and further in extracting information that provide certain specified features. Molecule the 
Signal \& Image Processing : An International Journal (SIPIJ) Vol.3, No.4, August 2012

$p$-azoxyanisole with chemical formula $\mathrm{C}_{14} \mathrm{~N}_{2} \mathrm{O}_{3} \mathrm{H}_{14}$ that is organic, white and aromatic compound in solid state exhibiting liquid crystalline is shown in figure 1.

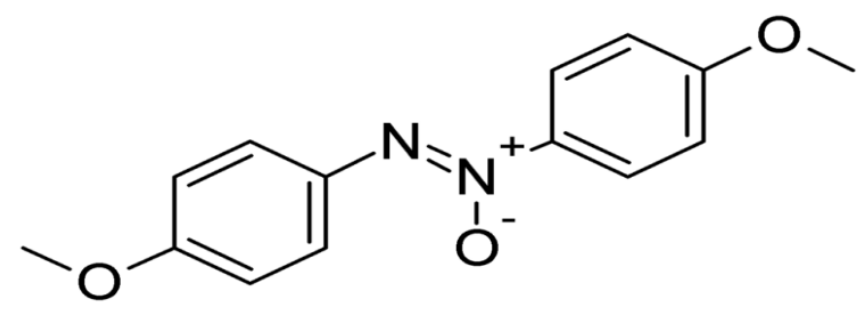

Figure 1: Molecular Structure of $p$-azoxyanisole

\section{MATERIALS AND METHODS}

Liquid crystal the $p$-azoxyanisole is purchased from Sigma Aldrich and used as such without any purification. Texture studies are performed with optical microscope Olympus BX-51 fitted with heating stage at the rate of $1^{\circ} \mathrm{C} /$ minute. Textures are obtained with slide is prepared rubbing glass plate in only one specified direction and sample is placed on it .This sample is placed on heating stage for observation with transparent cover slip over it. The sample is subjected to repeated scans for its thermal stability.

Quantitative analysis of textures is realized with numerous textures of entire liquid crystalline material in the entire process of heating and cooling and extraction of data by processing them. The correlation is established between two methods viz with texture measurements performed by optical microscopy and data obtained from the textures obtained by processing the image with MATLAB software. These textures are arranged sequentially according to temperature both in heating and cooling. By using MATLAB 7.2 the images are resized into 128X128 and converted into gray scale images and by using MATLAB code for computing the GLCM features. Studies are performed with illustrative graphs between various physical quantities labeled as parameter and temperatures responsible for its formation.

\section{RESULTS}

Texture obtained by liquid crystal $p$-azoxyanisole [12-13] is monotropically nematic i.e. exhibiting same texture in both heating and cooling in nature with all the molecules oriented in specified direction where the center of gravity of molecule coincides with centre of symmetry. These nematic phases are characterized with first order transition [14]. As the relative positions each pixels are identical statistical approach is realized. Analysis of texture is performed with its distribution of its gray values at each and every point of image and computes certain set of statistical features that describe the texture. Statistical type of procedure in analysis is applicable when the texture primitives are comparable with pixel sizes of first order. This type of analysis explores the texture features [15-19] like energy, entropy, auto correlation, contrast, correlation, cluster prominence, dissimilarity, homogeneity, variance, sum average, sum variance, difference variance, sum and difference entropy. These extracted features are expressed as function of temperature. The significant features of textures are illustrated in compact way labeling as parameter on $\mathrm{Y}$ axis that alter with temperature on $\mathrm{X}$ axis as illustrated from figures 3 to figure 7 along with their variations. The results of analysis by textures are attributed graphically with 
Signal \& Image Processing : An International Journal (SIPIJ) Vol.3, No.4, August 2012

sufficient explanation and relationships that lead to liquid crystalline nature of nematic. Processing of textures in the experiment was conducted with database of 91 images collected for entire liquid crystal both in heating and cooling and an accuracy of $100 \%$ is retrieved. An illustrative texture of its representation is indicated in figure 2 in cooling process.

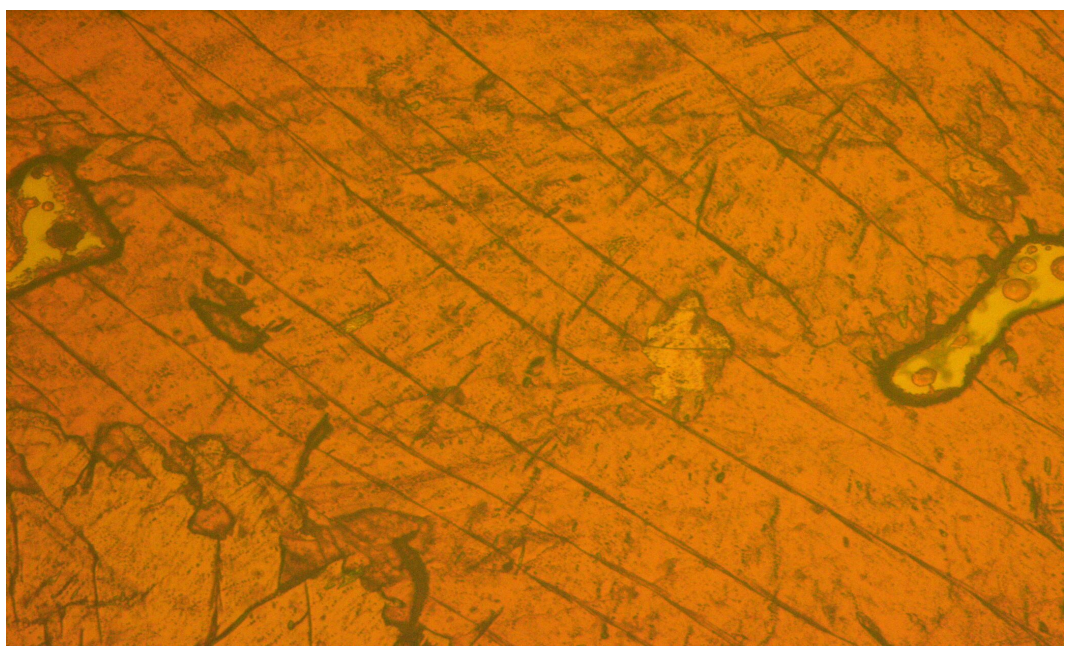

Figure 2: Illustrative nematic texture

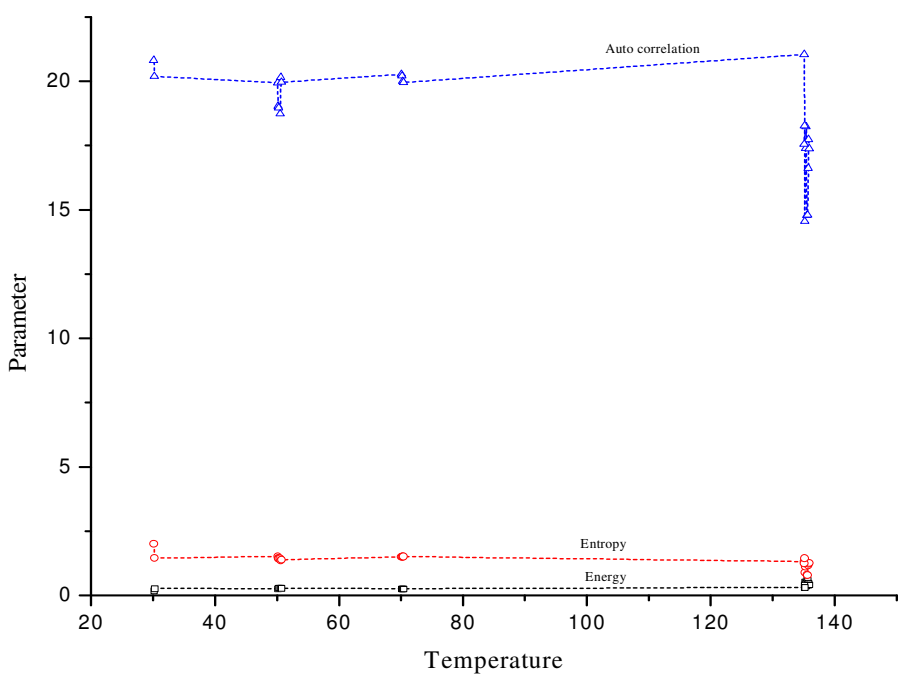

Figure 3: Illustration of Auto correlation, Energy and Entropy 
Signal \& Image Processing : An International Journal (SIPIJ) Vol.3, No.4, August 2012

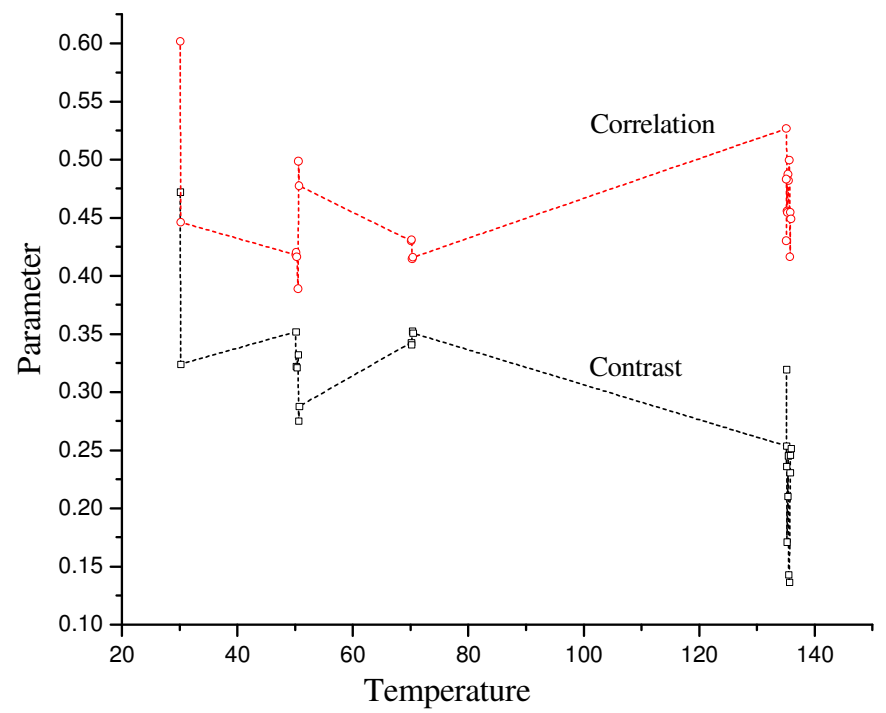

Figure 4: Illustration of Correlation and Contrast

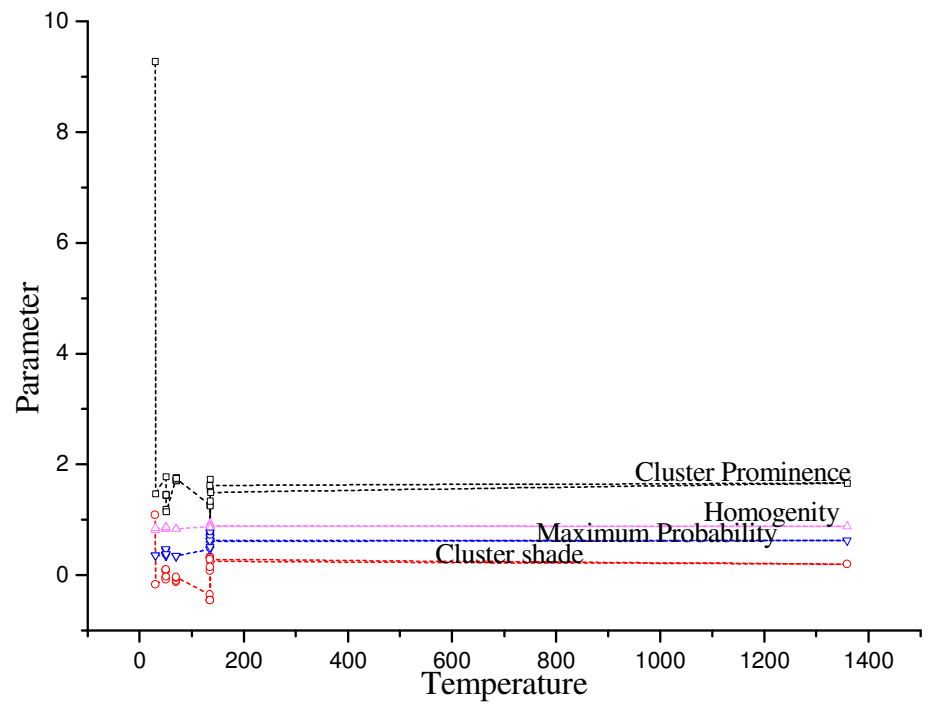

Figure 5: Illustration of Cluster variance, Homogeneity, Maximum probability and Cluster shade 
Signal \& Image Processing : An International Journal (SIPIJ) Vol.3, No.4, August 2012

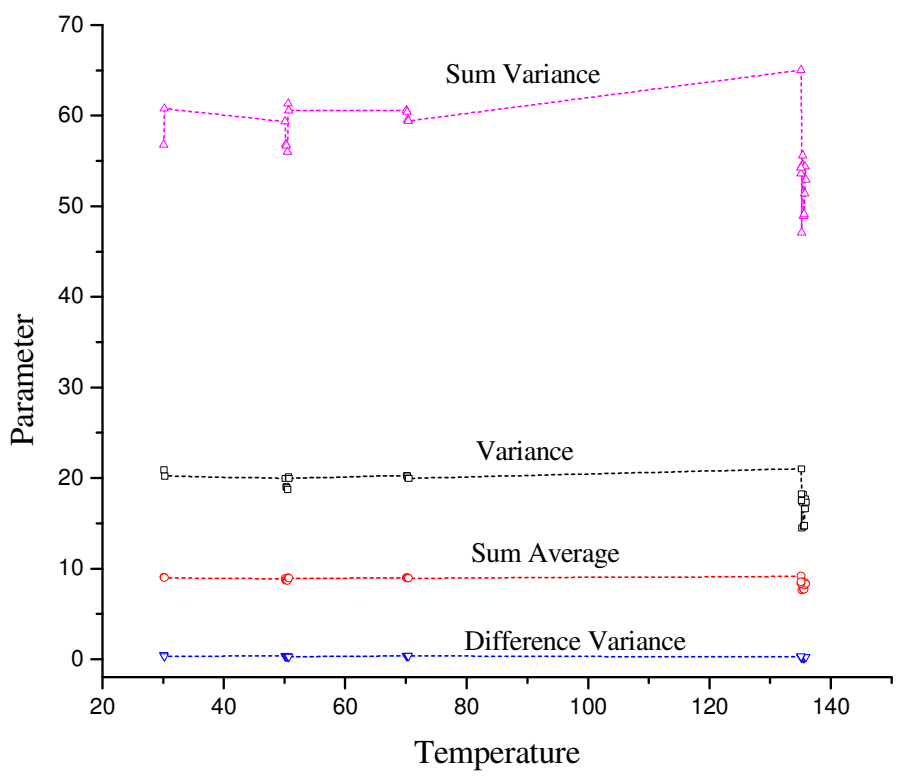

Figure.6: Illustration of Sum variance, variance, Sum Average and Difference variance

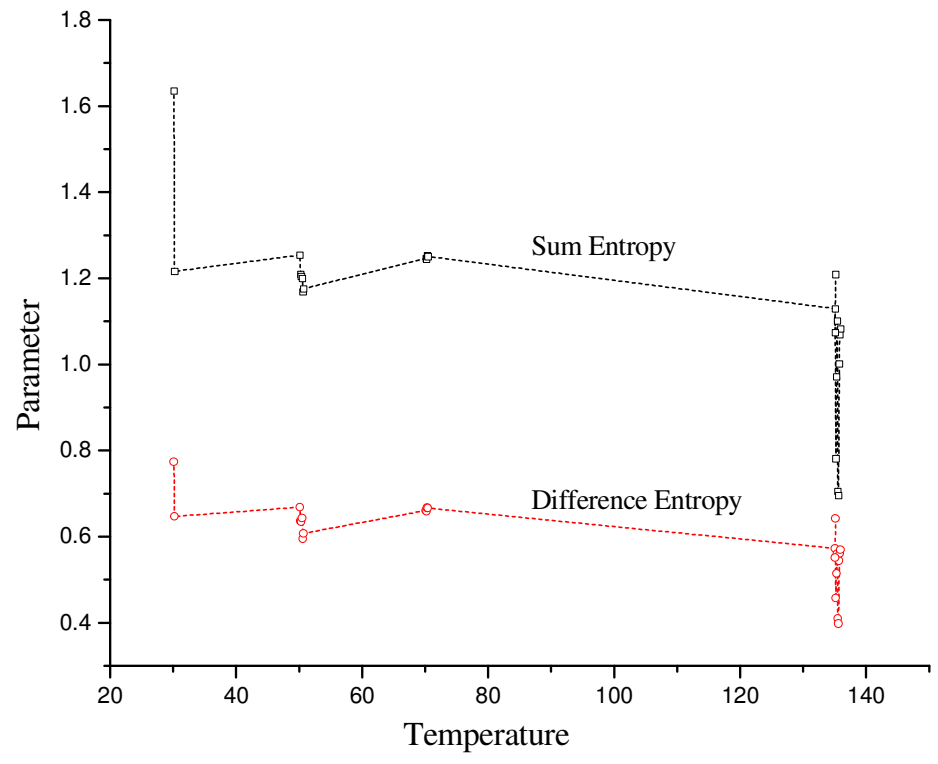

Figure 7: Illustration of Sum and Difference Entropy 
Signal \& Image Processing : An International Journal (SIPIJ) Vol.3, No.4, August 2012

\section{DISCUSSION}

A number of features from textures are obtained by the following equations with number of gray levels as $\mathrm{G}$ and $\mu$ 's are the means of $\mathrm{P}$ representing the matrix elements corresponding to the pixels $\mathrm{x}$ and $\mathrm{y}$. The following 15parameters are attributed along with relevant expressions. Entropy which measures randomness that is scalar quantity representing on grayscale image that gives statistical measure of randomness used to characterize the texture of the input image defined as

Entropy $=-\sum_{i=0}^{G-1} \sum_{j=0}^{G-1} P(i, j) \times \log [P(i, j)]$

Entropy of local attributes measures the predictability of a region that correspond to pixel intensities corresponding to a piecewise constant region. For entropy characterized with lower value is predictable further in statistical measurement its sum and difference is calculated by the relation

Sum Entropy $=-\sum_{i=0}^{2 G-2} P_{x+y}(i) \log P_{x+y}(i)$

Difference entropy $=-\sum_{i=0}^{G-1} P_{x+y}(i) \log \left(P_{x+y}(i)\right)$

The parameters responsible for the formation of texture characterized with low values of energy and entropy indicate the predictable textures are illustrated in figure 3.

Texture features such as contrast contribute to the determination of visual salience and thus can act to guide the selection process. A feature of the texture is that the intensity of each pixel gradually decreases before reaching to its isotropic state that has different patterns .A feature of study is that the even odd effect is observed between correlation and contrast as illustrated in figure 4.

Correlation analysis presents unique way of understanding the variables such as entropy and energy with precise attribute

Correlation $=\sum_{i=0}^{G-1} \Sigma_{j=0}^{G-1}[i \times j] \times \log \{P(i, j)]$

Autocorrelation is the cross correlation of a intensity of pixel with itself that provide similarity between the observations as function of temperature that provide repeating of patterns that remain unaltered.

For a given texture of embedded in a surrounding background of similar texture the perceived contrast of the texture depends substantially on the contrast of the background further texturecontrast illusion that does not involve eye movements.

A measure of contrast or local intensity variation will favour contributions from $\mathrm{P}(\mathrm{i}, \mathrm{j})$ away from the diagonal elements with its representation in matrix form by relation

Contrast $=\sum_{n=0}^{G-1} n 2\left\{\sum_{i=1}^{G} \sum_{j=1}^{G} P(i, j)\right\} \quad|\mathrm{i}-\mathrm{j}|=\mathrm{n}$ 
Signal \& Image Processing : An International Journal (SIPIJ) Vol.3, No.4, August 2012

Sum average of given texture it is required to quantify intuitive qualities like rough, smooth etc as a function of the spatial variation in pixel intensities which is performed by obtaining standard deviation of pixels in a neighborhood with corresponding relation

Sum Average $=\sum_{i=0}^{2 E_{0}} i P_{x+y}(i)$

Certain interesting features like shading, disappearance of pixels results in texture of resulting in reduced intensity of pixels that result in cluster Shade represented by

$$
\sum_{i=0}^{G-1} \sum_{j=0}^{G-1}\left\{i+j-\mu_{x}-\mu_{y}\right\}^{3} \times \mathrm{P}\{i, j\}
$$

The local bright and dark regions that favor the texture is characterized with cluster prominence with $\sum_{i=0}^{G-1} \sum_{j=0}^{G-1}\left\{i+j-\mu_{x}-\mu_{y}\right\}^{4} \times P\{i, j\}$

The statistical attributes of the texture further provide quantities leading to

Variance $=\sum_{i=0}^{G-1} \sum_{j=0}^{G-1}(i-\mu)^{2} P(i, j)$.

Homogenity $=\sum_{i=0}^{G-1} \sum_{j=0}^{G-1}\{P(i, j))^{2}$

The parameters of figure 5 reflect the local significance of the texture in comparison with its nearest neighborhood within the limit of 2 units. The parameter sum variance greater by 60 units with its difference is linear and its variance and sum average differed by 10 units is illustrated in figure 6. At the conclusive study the parameters change in similar manner with sum entropy[20] predominant that the difference by 0.8 as illustrated in figure 7 .

\section{CONCLUSIONS}

An attempt is made with framework for the examining the texture at different temperatures at every pixel of texture locally and its neighborhood points. Texture is analyzed with MATLAB by processing the image that alter with temperature. Its primitives measured are comparable with pixel sizes of first order with statistical type of procedure applicable in analysis. All significant features vary with temperature indicating the liquid crystalline nature of the material involving transitions from crystalline-nematic -isotropic and vice versa. Averaging of significant parameters with temperature can improve as contrast of texture. Studies of random texture reveal interesting features like cluster shade and cluster prominence that emphasize locally shadowed areas, homogeneity is almost an inverse function of energy associated with texture. The analysis of autocorrelation indicates the fineness of texture is same from its variation with temperature.

\section{ACKNOWLEDGEMENTS}

The author thanks the management of the GITAM University for its Support in providing academic environment. The corresponding author thanks Spectroscopy / Analytical Test Facility, Entrepreneurship centre, Society for Innovation \& Development (SID), Indian Institute of Science, Bangalore for providing Polarizing optical microscopic. 
Signal \& Image Processing : An International Journal (SIPIJ) Vol.3, No.4, August 2012

\section{REFERENCES}

[1] C.M. Paleos, D. Tsiourvos, "Supramolecular Hydrogen Bonded Liquid Crystals" Liquid Crystals (2001) Volume 28 No.8 ,pp1127-1161.

[2] Kato, in: D. Demus, G. Goodby, W. Gray, H.W. Spiess, V.W. Vill (Eds.) (1998) Hand Book of Liquid crystals, Wiely-VCH.

[3] G.W. Gray, and J.W.G. Good By Smectic Liquid Crystals (1984) Leonard Hill Press, Glassgow and London

[4] Iam Choon Khoo, Francesco Simoni, Physics of Liquid Crystal Materials(1991) Gordon and Breach Science Publishers.

[5] Oge Marques , Practical Image and Video Processing Using MATLAB(2011) John Wiley \& Sons Publishers.

[6] Gonzalez Digital Image Processing (1993) 2nd Ed, Pearson Education India Publishers

[7] Anil .K. Jain , Fundamentals of Digital Image Processing (1989) Prentice Hall Publishers

[8] Hazem M Raafat, Andrew K.C Wong, (1988) "A texture information-directed region growing algorithm for image segmentation and region classification" Computer Vision Graphics and Image Processing Vol.43, Issue 1, pp 1-28

[9] J Sklansky(1978) "Image segmentation and feature extraction" IEEE transaction on Systems, Man and Cybernetics, Vol 8,No 4 pp 237-247

[10] A.Rosenfeld, Editor (1976) Digital Picture Analysis, Springer Verlag, Berlin Publishers

[11] Ojala, T. and M Pietikäinen (2004) Texture Classification. Machine Vision and Media Processing Unit, University of Oulu, Finland. Available at ttp://homepages.inf.ed.ac.uk/rbf/CVonline/LOCAL_COPIES/OJALA1/ texclas.htm

[12] S. Chandrasekhar and N.V.Madhusudana (1973) "Nematic Order in p-azoxyanisole and its Dependence on Pressure, Volume and Temperature" Molecular Crystals and Liquid Crystals Vol. 24, No 1-2, pp. 179-186

[13] S. Chandrasekhar and N.V.Madhusudana (1969) " Orientational order in p- Azoxyanisole ,pAzoxyphenetole and their mixtures in the Nematic phase" J. Phys. Colloques Vol 30 No 4 ppC4-24 C4-27

[14] K. Takashi, S. Kohmota, M. Yamamoto and K. Kishikawa (2004) "Liquid crystalline amides: linear arrangement of rod-like molecules by lateral intermolecular hydrogen bonding and molecular shape effect" Journal of Material Chemistry Vol 14, No 23 ,pp 3449 -3456

[15] R.M. Haralick, K. Shanmugam, and I. Dinstein(1973) "Texural features for Image Classification" IEEE Trans. on Systems, Man and Cybernetics, Vol. SMC-3, No 6,pp. 610-621

[16] R.W. Conners, M.M. Trivedi, and C.A. Harlow(1984) "Segmentation of a high-resolution urban scene using texture operators" Computer Vision, Graphics, and Image Processing, Vol. 25, No3,pp. 273- 310 . 
Signal \& Image Processing : An International Journal (SIPIJ) Vol.3, No.4, August 2012

[17] Yuan Been Chen and Oscal T.-C. Chen (2009)"Image Segmentation Method Using Thresholds Automatically Determined from Picture Contents"EURASIP Journal on Image and Video Processing Vol.2009, Article ID 140492, pp 1-15

[18] Yunyun Yang and Boying Wu (2012) "Fast multiphase image segmentation model for images with inhomogeneity" J.Electron Imaging Vol 21,013015 J. Electron. Imaging 21, 013015 (29 March 2012); http://dx.doi.org/10.1117/1.JEI.21.1.013015

[19] Salim Ben Chaabane, Mounir Sayadi, Farhat Fnaiech and Eric Brassart (2010) "Colour Image Segmentation Using Homogeneity Method and Data Fusion Techniques" EURASIP Journal on Advances in Signal Processing Vol. 2010, Article ID 367297 pp 1-11

[20] Andre L. Barbieri , G.F. de Arruda , Francisco A. Rodrigues , "Odemir M. Bruno ,and Luciano da Fontoura Costa "An entropy-based approach to automatic image segmentation of satellite images" (2011) Physica A,Vol 390 No 3 pp512-618

\section{Authors}

Corresponding Author Dr.Ravi Shankar Kumar.Ch is associated with frontier areas of research in Materials science and published 12 numbers of papers in national and International journals as corresponding author and further with 23 papers published in conferences of national and International repute.

The author is reviewer of peer reviewed journals and member of various academic and professional bodies and peer teams.

Currently the author is working in implementing the materials and design of display devices electronically further in computational work of textures or images. The textures/images obtained are processed by using Image processing techniques using MATLAB in studying its features and confirming with obtained texture.

Ratna Raju M. is currently associated with research for his doctoral work with image processing techniques in its confirmation and retrieval in computing gray level coocurence features of images.
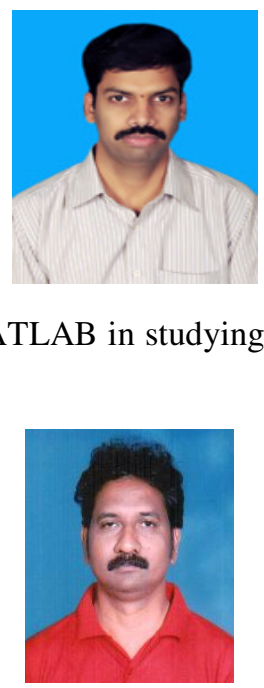